\title{
Chemical evolution of galaxies and galaxy formation mechanisms
}

\section{Francesca Matteucci ${ }^{* \dagger}$}

Astronomy Department, University of Trieste, Italy

E-mail: matteucci@ts.astro.it

\begin{abstract}
We will review the chemical evolution of galaxies of different morphological type: spirals, ellipticals and irregulars, and discuss how chemical abundances can constrain galaxy formation mechanisms. In particular, the following questions will be addressed: can the Milky Way galaxy be the result of merging of dwarf spheroidals? Are the characteristics of spheroids (ellipticals and bulges) more compatible with the monolithic or the hierarchical clustering scenario for galaxy formation? Are population III stars required to explain the chemical properties of stellar populations in galaxies? All of the answers to the previous questions will be negative, thus imposing clear constraints on galaxy formation mechanisms.
\end{abstract}

Baryons in Dark Matter Halos

5-9 October 2004

Novigrad, Croatia

\footnotetext{
${ }^{*}$ Speaker.

${ }^{\dagger}$ A footnote may follow.
} 


\section{Introduction}

The chemical evolution of galaxies studies the evolution of the abundances of the most common chemical elements in the gas in galaxies. The main ingredients required to build models of galactic chemical evolution are:

- The initial conditions of the gas out of which the first stars will form; therefore, whether the gas is primordial (no metals) or already enriched in heavy elements by a pregalactic stellar generation of very massive stars (population III stars).

- The birthrate function which describes the history of star formation and is one of the most crucial parameters in galaxy evolution. Generally, for the sake of simplicity this function is parametrized in the following way:

$$
B(m, t)=\psi(t) \varphi(m)
$$

where:

$$
\psi(t)=S F R
$$

is the so-called star formation rate (SFR). Normally the SFR is assumed to be proportional to some power of the gas density (Schmidt, 1959) through a constant parameter called the efficiency of star formation, $v$, which has the dimensions of time $^{-1}$ and where:

$$
\varphi(m)=I M F
$$

is the so-called initial mass function (IMF). The IMF is assumed to be a power law with one or more slopes (see Scalo, 1986;1998 for reviews).

- Stellar nucleosynthesis, namely how much and when stars eject their newly produced elements in the interstellar medium (ISM).

- Supplementary but not less important parameters: gas accretion and gas outflow from galaxies.

In this paper we will describe the results obtained by detailed chemical evolution models following the evolution in space and time of several chemical elemets $(\mathrm{H}, \mathrm{He}, \mathrm{D}, \mathrm{C}, \mathrm{N}, \mathrm{O}, \alpha$-elements, $\mathrm{Fe}$ ) in galaxies of different morphological type, in particular in spirals (the Milky Way), ellipticals and irregulars. The intent will be to compare the model predictions with observational data and infer constraints on galaxy formation mechanisms for all of these galaxies. In fact, two competing scenarios for galaxy formation have confronted each other in the past few years. One is the cosmologically based scenario of hierarchical formation of galaxies, where it is assumed that like for the dark matter halos also the baryons should have been assembled hierarchically. In other words, the small galaxies formed first and then successive mergers of these small objects gave rise to the more massive ones. In this picture galaxies form at all epochs. The other scenario is not included in any cosmological context and it simply assumes that most of the galaxies started forming stars at early epochs and then evolved with different star formation rates implying that some objects such as the spheroids which contain only old stars, suffered a quite intense star formation and consumed most of their original gas. On the other hand, other galaxies such as spirals and irregulars evolved with a slower star formation rate and they have still an active star formation at the present time. This scenario is justified by the simple fact that most of the galaxies contain stars as old as the universe. For 
historical reasons, we will refer to this latter scenario as the monolithic or high-redshift formation one.

\section{Stellar yields and SN progenitors}

To build a chemical evolution model we need to specify the stellar yields, namely the amount of material that each star can produce and eject into the ISM in the form of a given element.

We divide the stars in several mass ranges, in particular:

-Low and Intermediate mass stars $\left(0.8 \leq M / M_{\odot} \leq 8.0\right)$ produce ${ }^{4} \mathrm{He}, \mathrm{C}, \mathrm{N}$, s-process $(A>$ 90) elements, which eject into the ISM during the formation of a planetary nebula. The yield calculations that we will adopt in the following are from van den Hoeck \& Groenewegen (1997). Other calculations can be found in Marigo et al. (1996), Meynet \& Maeder (2002), Ventura et al. (2002), Siess et al. (2002).

-Massive stars $\left(M \geq 10 M_{\odot}\right)$ produce mainly $\alpha$-elements $(\mathrm{O}, \mathrm{Ne}, \mathrm{Mg}, \mathrm{Si}, \mathrm{S}, \mathrm{Ca})$, some $\mathrm{Fe}$ peak elements, s-process elements $(A<90)$ and r-process elements. They explode as core-collapse SNe. We will adopt the yields from Woosley \& Weaver (1995) or Thielemann et al. (1996). Other studies of this kind can be found in Langer \& Henkel (1995), Nomoto et al. (1997), Rauscher et al. (2002).

-Type Ia SNe are assumed to be C-O white dwarfs in binary systems exploding by C-deflagration after reaching the Chandrasekhar mass. They mainly produce $\mathrm{Fe}$ and Fe-peak elements. The yields we will adopt are from Nomoto et al. (1997), model W7.

-Very massive objects $\left(M>100 M_{\odot}\right.$, pop III). These stars, if exist, explode as a consequence of pair-creation instability. The yields adopted are from Umeda \& Nomoto (2001), Heger \& Woosley (2002) and Chieffi \& Limongi (2004).

\subsection{Type Ia SN rates}

Two main scenarios have been proposed in the last years for the progenitors of type Ia SNe and they are: a) The single degenerate scenario: the classical scenario of Whelan and Iben (1973), namely C-deflagration in a C-O WD reaching $M_{C h}$ after accreting material from a companion (RG). The clock for the explosion is given by the lifetime of the secondary star. The minimum timescale for the first type Ia SN to occur is therefore $t_{S N I a_{m i n}}=0.03 \mathrm{Gyr}$, which corresponds to a binary system formed by two $8 M_{\odot}$ stars (Greggio and Renzini 1983a).

b) The Double Degenerate scenario: the merging of two C-O WDs, due to gravitational wave radiation, which explode by C-deflagration when $M_{C h}$ is reached (Iben and Tutukov 1984; 1985). The minimum timescale for the explosion is given by the lifetime of the secondary star plus the gravitational time-delay $t_{S N I a_{\text {min }}}=0.03+\Delta t_{\text {grav }}=0.03+0.15$ Gyr (Tornambè \& Matteucci 1986).

c) A more recent model by Hachisu et al. (1999) is based on the single degenerate scenario but with a metallicity effect implying no type Ia systems can form for $[\mathrm{Fe} / \mathrm{H}]<-1.0$. This in turn implies a minimum timescale for type Ia SNe of $t_{S N I a_{m i n}}=0.33 \mathrm{Gyr}$ plus the metallicity time delay depending on the adopted chemical evolution code. 


\section{The chemical evolution of the Milky Way}

The Milky Way contains four main stellar population components: the halo, the thick-disk, the thin-disk and the bulge. The stars in the halo and the bulge are all old but those in the halo are only metal poor, whereas in the bulge there are also metal rich stars with over solar metallicity (Mc William \& Rich 1994). The stars in the thin disk are increasingly metal rich and the stars in the thick disk have metallicities intermediate between those of the halo and those of the thin disk. However, there is overlapping between the metallicities of stars in the halo and thick disk and those in the thick and thin disk. In these cases the stars in the various components can be distinguished by their different kinematical properties. The chemical and kinematical properties can give us indication about the mechanism of formation of the various galactic components. Models for the chemical evolution of the Milky Way presented in the past years can be summarized as follows:

\section{- Serial Formation}

In this scheme the halo, thick and thin disk form in sequence as a continuous collapse process (e.g. Matteucci \& François 1989), where the chemical enrichment proceeds sequentially and no overlapping between abundances in the stars of the different components, at variance with observations.

\section{- Parallel Formation}

The various Galactic components start forming at the same time and from the same gas but evolve at different rates, thus producing overlapping of the abundances of stars belonging to the different components (e.g. Pardi, Ferrini \& Matteucci 1995).

\section{- Two-Infall Formation}

The halo and disk form out of two separate episodes of infall of extragalactic gas almost completely independent from each other. Overlapping in metallicity is also predicted (e.g. Chiappini, Matteucci \& Gratton 1997; Chang et al. 1999).

\section{- Inhomogeneous Formation}

In the early halo phases, for $[\mathrm{Fe} / \mathrm{H}]<-3.0$, mixing was not efficient thus implying pollution from single SNe (Tsujimoto et al. 1999; Argast et al. 2000; Oey 2000). In this case a large spread predicted for $[\mathrm{Fe} / \mathrm{H}]<-3.0$.

\subsection{The time-delay model}

The difference in the timescales for galactic chemical enrichment of SNII and Ia produce a time-delay in the Fe production relative to $\alpha$-elements (Tinsley 1979; Greggio \& Renzini 1983b; Matteucci 1986). In Figure 1 are shown the predictions of the two-infall model for the [O/Fe] versus $[\mathrm{Fe} / \mathrm{H}]$ in the case where both SNIa and SNII are contributing to the Fe enrichment in the proportions of $2 / 3$ for type Ia $\mathrm{SNe}$ and of $1 / 3$ for type II SNe as well as two euristic cases where $\mathrm{Fe}$ is assumed to be produced only in type Ia SNe (upper curve) and only in type II SNe. Both these cases cannot produce a good fit to the data, whereas the mixed case gives a very good fit. The time-delay model interpretation is presently the most accepted interpretation of the $[\mathrm{X} / \mathrm{Fe}]$ versus $[\mathrm{Fe} / \mathrm{H}]$ plots. The time at which the change in slope occurs in the $[\mathrm{O} / \mathrm{Fe}]$ corresponds to the time at which a substantial number of type Ia SNe have exploded so to induce an increase in Fe production due to type Ia SNe. This timescale represents also the duration of the halo-thick-disk phase and is 1.5 - 2.0 Gyr (Matteucci \& Greggio, 1986; Matteucci \& François, 1989). 

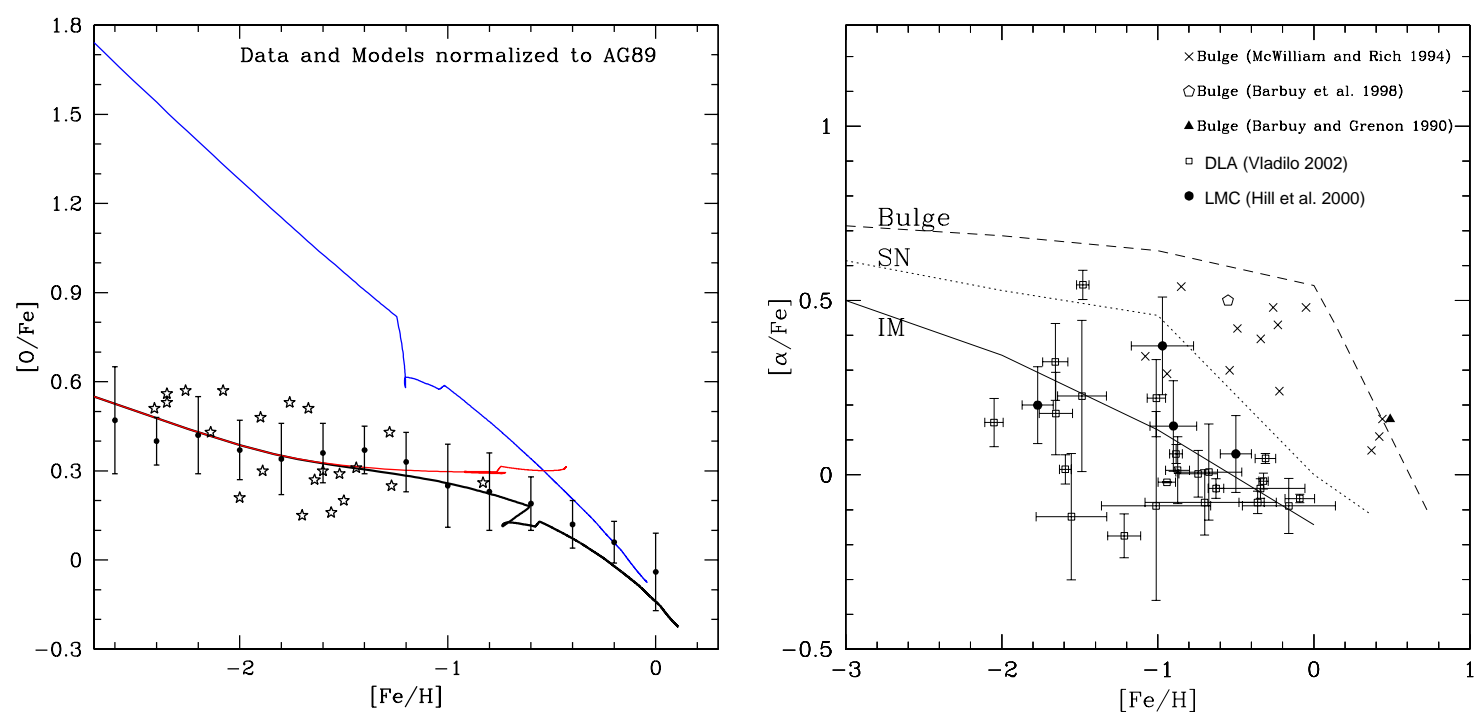

Figure 1: Left panel: predicted and observed $[\mathrm{O} / \mathrm{Fe}]$ vs. $[\mathrm{Fe} / \mathrm{H}]$ for the solar neighbourhood. The upper curve refers to a model where all the solar Fe is attributed to the type Ia SNe, whereas the fht curve refers to a model where all the solar Fe is attributed to type II SNe. The best model is the one which contains contributions from both SN types (see text) and demonstrates the goodness of the time-delay model interpretation. The data are from Melendez \& Barbuy (2002) and are normalized, as well as the predictions, to the solar abundances of Anders \& Grevesse (1989). Right panel: predicted $[\alpha / \mathrm{Fe}]$ ratios in galaxies of different morphological type. The dashed curve refers to the galactic bulge, the dotted curve refers to the solar vicinity and the continuous one to an irregular magellanic galaxy. The data sources are shown in the fi gure.

One conclusion, common to most chemical evolution models for the solar vicinity concerns the fit of the observed metallicity distribution for the G-dwarfs of the thin-disk. In particular, it is suggested that the best fit to such distribution is obtained by assuming a quite long timescale for the thin-disk formation (Chiappini et al. 1997). The conclusion that the galactic disk at the solar circle should have assembled slowly on a timescale of 6-8 Gyr was reached later also by the following authors: Boissier and Prantzos (1999), Chang et al. (1999) and Alibès et al. (2001).

Some common conclusions concerning the galactic disk refer to the abundance, star formation rate and gas gradients. In order to fit these gradients the galactic disk should have assembled insideout in the sense that the internal regions should have collapsed more quickly than the external ones and the assumed star formation rate should be a strongly varying function of the galactocentric distance (Matteucci \& François 1989; Chiappini et al. 1997,2001; Goswami \& Prantzos 2000; Alibes et al. 2001). Moreover, an inside-out formation of the Galaxy is suggested by the fact that the globular clusters of the inner halo are coeval ( $\Delta$ (age) $\sim 0.5 \mathrm{Gyr}$, Rosenberg et al. 1999), whereas the age difference seems to increase in the outer halo (2-4 Gyr, Lee et al. 1994).

Concerning the galactic bulge, there have been several suggestions about its formation and evolution here summarized:

i) Accretion of extant stellar systems which eventually settle in the center of the Galaxy.

ii) Accumulation of gas at the center of the Galaxy and subsequent evolution with either fast 
or slow SF.

iii) Accumulation either rapid or slow of metal enriched gas from the halo or thick disk in the Galaxy center.

iv) The Bulge formed out of inflow of metal enriched gas from the thin-disk.

Numerical experiments (Matteucci \& Brocato 1990; Matteucci et al. 1999) have shown that the metallicity distribution of stars in the bulge (Mc William \& Rich 1994) suggests the Bulge formed in only $\sim 0.5 \mathrm{Gyr}$ contemporarily to the halo formation. This conclusion is supported by the finding of Wyse \& Gilmore (1992) that the distribution of the stellar angular momentum per unit mass in the bulge and the halo are very similar and by the overabundance of $\alpha$-elements extended over a large range of $[\mathrm{Fe} / \mathrm{H}]$.

\section{The delay-model in galaxies of different morphological type}

The behaviour of abundance ratios versus metallicity (normally $[\mathrm{Fe} / \mathrm{H}]$ ) depends not only upon stellar nucleosynthesis but also upon the assumed star formation history for a given galaxy. In Figure 1 we show the different predictions for the galactic bulge (upper curve) or a spheroidal system in general, for the solar neighbourhood and for a Magellanic irregular galaxy (e.g. LMC, SMC). The only difference in the various models are the star formation rates $\psi(t)$ : in the bulge the efficiency of the SFR is quite high $\left(v=20 G y r^{-1}\right)$, in the solar neighbourhood is $v=1 G y r^{-1}$ and in the irregular galaxy is $v=0.1 G y r^{-1}$. As one can see, a fast SFR produces a long plateau in the $[\mathrm{O} / \mathrm{Fe}]$ ratio and a change of slope occurring only at solar $[\mathrm{Fe} / \mathrm{H}]$. This is due to the fact the $[\mathrm{Fe} / \mathrm{H}]$ increases very fast due only to the contribution of type II SNe, then when the first type Ia $\mathrm{SNe}$ occur and the slope changes the attained metal content is quite high. The contrary happens in systems with a slow SFR, where at the appearence of the type Ia SNe the metallicity is still quite low due to the slow increase of the Fe abundance. This fact is important since it allows us to distinguish among different objects only by looking at their abundance pattern. In this way we can, in fact, identify the nature of the high redshift objects. In the figure 1, right panel, the LMC data as well as the data for Damped-Lyman $\alpha$ (DLA) systems are shown and the comparison indicates that they can be identified only with irregular systems. Data for the galactic bulge are also shown and they agree with our predictions.

\section{Spiral Galaxies}

Here we summarize the most important conclusions on spirals in general. In particular, we refer to an interesting study by Boissier et al. (2001) who studied a sample of normal spiral galaxies extensively described in Boselli et al.(1996) and found that:

i) Massive disks are redder than low mass ones.

ii) Massive disks are more metal rich and less gas rich than low mass ones.

iii) Massive disks have similar star formation efficiency than low mass ones.

All of these properties taken together imply that massive disks are older than low mass ones, at variance with the predictions of the hierarchical clustering scenario. 


\section{Elliptical Galaxies}

In the past years several mechanisms for the formation of elliptical galaxies have been put forward, in particular:

1) An early monolithic collapse of a gas cloud or early merging of lumps of gas where dissipation plays a fundamental role (Larson 1974; Arimoto \& Yoshii 1987; Matteucci \& Tornambè 1987). In this picture the star formation stops after the occurrence of the galactic wind.

2) Bursts of star formation in merging subsystems made of gas (Tinsley \& Larson 1979). In this picture SF stops after the last burst and gas is lost via stripping or galactic wind.

3) Early merging of lumps containing gas and stars in which some dissipation is present (Bender et al. 1993).

4) Merging of early formed stellar systems in a wide redshift range and preferentially at late epochs (Kauffmann \& White, 1993). A burst of star formation can occur during the major merging where $\sim 30 \%$ of the stars can be formed (Kauffmann 1996). In this picture, massive ellipticals form last and elliptical formation occurs on a large range of redshifts. For a more recent point of view see also Steinmetz (2003).

\subsection{Arguments in favor of the high-z elliptical formation}

There are substantially two points of view: ellipticals formed at high redshift and evolved passively since then or ellipticals formed in a large redshift range. The arguments in favor of the first hypothesis are:

1) the tightness of the color-central velocity dispersion relation found for Virgo and Coma galaxies (Bower et al. 1992). The argument goes like that: from the observed color scatter one can infer that $t_{H}-t_{F} \sim 2 \mathrm{Gyr}\left(t_{H}\right.$ is the Hubble time and $t_{F}$ the time of galaxy formation). Therefore, if $t_{H}=15 \mathrm{Gyr}$ then the youngest ellipticals must have formed $\sim 13 \mathrm{Gyr}$ ago at $z \geq 2$.

2) The thinness of the fundamental plane for ellipticals in the same two clusters (Renzini \& Ciotti 1993).

3) The tightness of the color-magnitude relation for ellipticals in clusters up to $z \sim 1$ (Kodama et al. 1998; Stanford et al. 1998).

4) The finding that $\langle[\mathrm{Mg} / \mathrm{Fe}]\rangle_{*}>0$ in the nuclei of large ellipticals and that is increasing with $M_{\text {gal }}$ and luminosity (Worthey et al. 1992; Matteucci 1994; Jorgensen 1999; Kuntschner 2001), suggests, on the basis of the time-delay model, that larger ellipticals evolved faster than smaller ones. However, variation of the IMF cannot be excluded but they would be quite "ad hoc".

5) The existence of Lyman-break galaxies at $z \geq 3$, where the SFR is as high as $\sim 1000 M_{\odot} y r^{-1}$, can be the young ellipticals (e.g. Matteucci \& Pipino 2002).

6) Luminous star forming infrared galaxies found at $z>2$ suggesting that they are the progenitors of massive spheroidals (Daddi et al. 2004).

7) The existence of old fully assembled massive spheroidals already at $1.6 \leq z \leq 1.9$ (Cimatti et al. 2004).

\subsection{Arguments in favor of the formation of ellipticals at low redshift}

The main arguments in favor of a late formation of ellipticals are : 

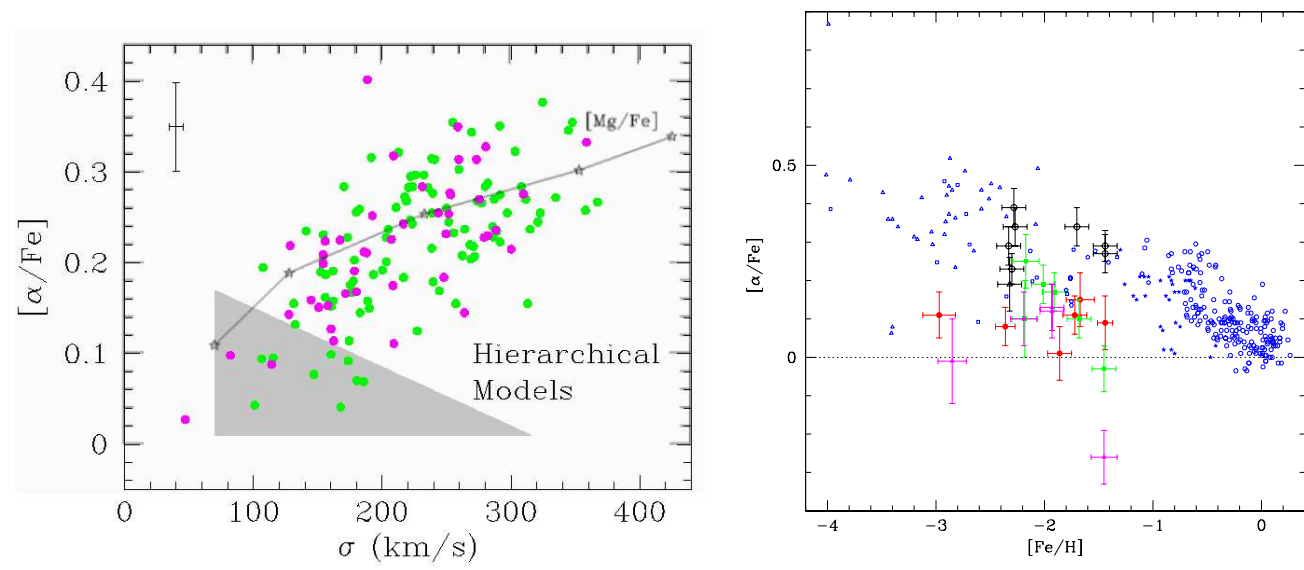

Figure 2: Left panel: the $[\alpha / \mathrm{Fe}]$ ratio in the dominant stellar population of ellipticals, as predicted by a semi-analytical hierarchical model and by a "monolithic" model. The data and the predictions from the semi-analytical model (the shaded area) are from Thomas et al. (2002), whereas the monolithic model results (continuous line) are from Pipino et al. (2002). Right panel: observed $[\alpha / \mathrm{Fe}]$ ratios in the solar neighbourhood and in dwarf speroidals of the local group (data with the error bars). Figure from Shetrone et al. (2001).

1) the relative large values of the $H_{\beta}$ index measured in a sample of nearby ellipticals which could indicate prolonged star formation activity up to 2 Gyr ago (Gonzalez 1993; Faber et al. 1995; Trager et al. 1998).

2) The tight relations in the fundamental plane are due to a conspiracy of age and metallicity in the sense that there exists an age-metallicity anticorrelation with the more metal rich galaxies being younger (Trager et al. 1998; Ferreras et al. 1999; Trager et al. 2000).

3) The apparent paucity of high luminosity ellipticals at $z \sim 1$ compared to now (Kauffmann et al. 1996; Zepf 1997; Franceschini et al. 1998).

\section{Models for Ellipticals}

Here we will compare the predictions concerning abundance ratios from a model where the ellipticals form at high redshift with high star formation rate and suffer early galactic winds (Pipino et al. 2002) with the predictions of a semy-analytical hierarchical model (Thomas et al. 2002). In Figure 2, left panel, we show the predicted $[\alpha / \mathrm{Fe}]$ vs. stellar velocity dispersion (galactic mass) of the two models compared with observational data It is clear from the figure that the early formation model can reproduce the increase of the $[\alpha / \mathrm{Fe}]$ ratio with galactic mass, whereas the predictions of the hierarchical models are at variance with the observations. This suggests that more massive ellipticals formed faster than less massive ones (Matteucci 1994, Matteucci et al. 1998).

\section{Dwarf Galaxies}

Dwarf Irregular (DIG) and Blue Compact (BCG) galaxies with low metallicity and high gas content are interesting objects for studying galaxy evolution. Dwarf spheroidals are similar to 
ellipticals: old stars and no gas. Dwarf galaxies in general are considered to be the building blocks of larger galaxies in the hierarchical clustering formation scenario. In particular, we will focuse on dwarf spheroidals (dSph) and discuss whether the local ones could have been the building blocks of the Milky Way. In Figure 2, right panel, we show the $[\alpha / \mathrm{Fe}]$ ratios in the stars in the solar vicinity and in the dwarf spheroidals of the local group. As one can see, the abundance ratios in dSphs show a quite different distribution relative to the solar neighbourhood one, resembling strictly the predicted case for an irregular galaxy shown in Figure 1, right panel. As explained before, the behaviour of the abundance ratios are dependent upon the star formation rate, thus suggesting that the dwarf spheroidals must have evolved more slowly than the solar region. Recent models for the dwarf spheroidal of the local group (Lanfranchi \& Matteucci 2003; 2004) have suggested that to reproduce the observed abundance pattern these galaxies must have evolved with a low SFR and suffered intense galactic winds. As a consequence of this, dSph galaxies cannot be the building blocks of a spiral galaxy such as the Milky Way.

\section{Population III Stars}

Both cosmological and stellar formation arguments (see Ferrara \& Salvaterra, 2004 for a recent review) suggest the possibility of a first stellar generation of very massive stars $\left(M>100 M_{\odot}\right)$ ending up their lives as pair-creation SNe. Chemical evolution models, adopting the most recent nucleosynthesis prescriptions for very massive stars, have shown that both in spheroids (Matteucci \& Pipino, 2005) and the Milky Way (Ballero et al., in preparation) the effect of Pop III stars on stellar abundances is negligible, if these very massive stars formed only until $Z_{t h} \leq 10^{-4} Z_{\odot}$, where $Z_{t h}$ is the threshold metallicity above which very massive stars should not form any more. On the other hand, if we allow the very massive stars to form for a period not shorter than $0.1 \mathrm{Gyr}$, then the predicted abundances are at variance with observational data both in the Milky Way and in ellipticals.

\section{References}

[1] Alibés, A., Labay, J., Canal, R. 2001, A\&A, 370, 1103

[2] Anders, E., Grevesse, N. 1989, Geochim. Cosmochim.Acta, 53, 197

[3] Argast, D., Samland, M., Gerhard, O.E., Thielemann, F.-K. 2000, A\&A, 356, 873

[4] Arimoto, N., Yoshii, Y., 1987, A\&A, 173, 23

[5] Barbuy, B., Grenon, M. 1990, in ESO/CTIO Workshop on Bulges of Galaxies, ESO Publ. p.83

[6] Barbuy, B., Ortolani, S., Bica, E. 1998, A\&AS, 132, 333

[7] Bender, R., Burstein, D., Faber, S.M., 1993, ApJ, 411, 153

[8] Boissier, S., Prantzos, N. 1999, MNRAS, 307, 857

[9] Boissier, S., Boselli, A., Prantzos, N., Gavazzi, G., 2001, MNRAS, 321, 733

[10] Boselli, A., Gavazzi, G., Donas, J., Scodeggio, M., 2001, AJ, 121, 753

[11] Bower, R.G., Lucey, J.R., ellis, R.S., 1992, MNRAS, 254, 601 
[12] Chang, R.X., Hou, J.L., Shu, C.G., Fu, C.Q. 1999, A\&A, 350, 38

[13] Chiappini, C., Matteucci, F., Gratton, R. 1997, ApJ, 477, 765

[14] Chiappini, C., Matteucci, F., Romano, D. 2001, ApJ, 554, 1044

[15] Chieffi , A., Limongi, M., 2004, ApJ, 608, 405

[16] Cimatti, A., Daddi, E., Renzini, A., et al., 2004, Nature, 430, 184

[17] Daddi, E., Cimatti, A., Renzini, A., et al., 2004, ApJ, 600,127

[18] Faber, S.M., Trager, S.C., Gonzalez, J.J., Worthey, G., 1995, IAU Symp. N.164, p.249

[19] Ferrara, A., Salvaterra, R., 2004, astro-ph/0406554

[20] Ferreras, I., Charlot, S., Silk, J., 1999, ApJ, 521, 81

[21] Franceschini, A., Silva, L., Fasano, G. et al., 1998, ApJ, 506, 600

[22] Gonzalez, J.J., 1993, PhD Thesis, University of California, Santa Cruz, Dissertation Abstracts International, Volume 54-05, Section B, p.2551

[23] Goswami, A., Prantzos, N. 2000, A\&A, 359, 191

[24] Greggio, L., Renzini, A. 1983a, A\&A, 118, 217

[25] Greggio, L., Renzini, A. 1983b in "The First Stellar Generations", Mem. Soc. Astron. It., Vol. 54, p.311

[26] Jorgensen, I., 1999, MNRAS, 306, 607

[27] Kauffmann, G., 1996, MNRAS, 281, 487

[28] Kauffmann, G., White, S.D.M., 1993, MNRAS, 261, 921

[29] Kauffmann, G., Charlot, S., White, S.D.M., 1996, MNRAS, 283, 117

[30] Kodama, T., Arimoto, N., Barger, A.J., Arag'on-Salamanca, A., 1998, A\&A, 334, 99

[31] Kuntschner, H., 2001, Ap\&SS, 276, 885

[32] Hachisu, I, Kato, M., Nomoto, K. 1999, ApJ, 522, 487

[33] Heger, X., Woosley, S.E., 2002, ApJ, 567, 532

[34] Hill, V., François, P., et al. 2000, A\&A, 364, L19

[35] Iben, I.Jr., Tutukov, A.V., 1984, ApJS 54, 335

[36] Lanfranchi, G., Matteucci, F., 2003, MNRAS, 345, 71

[37] Lanfranchi, G., Matteucci, F., 2004, MNRAS, 351, 1338

[38] Langer, N., Henkel, C. 1995, Space Sci. Rev. 74, 343

[39] Larson, R.B., 1974, MNRAS, 169, 229

[40] Lee, Y.W., Demarque, P., Zinn, R., 1994, ApJ, 423, 248

[41] Matteucci, F. 1986, ApJ, 305, L81

[42] Matteucci, F., 1994, A\&A, 288, 57

[43] Matteucci, F., Brocato, E. 1990, ApJ, 365, 539 
[44] Matteucci, F., François, P. 1989, MNRAS, 239, 885

[45] Matteucci, F., Greggio, L. 1986, A\&A 154, 279

[46] Matteucci, F., Pipino, A. 2002, ApJ, 569, L69

[47] Matteucci, F., Pipino, A., 2005, MNRAS, in press

[48] Matteucci, F., Ponzone, R., Gibson, B.K., 1999, A\&A, 335, 855

[49] Matteucci, F., Tornambé, A., 1987, A\&A, 185, 51

[50] Marigo, P., Bressan, A., Chiosi, C. 1996, A\&A, 313, 545

[51] McWilliam, A., Rich, R.M. 1994, ApJS, 91, 7

[52] Mc Willliam, A., Rich, R.M., 2004, Carnegie Observatories Astrophysics Series, Vol. 4: Origin and Evolution of the Elements, 2004

[53] Melendez, J., Barbuy, B. 2002, ApJ, 575, 474

[54] Meynet, G., Maeder, A. 2002, A\&A, 390, 561

[55] Nomoto, K., Iwamoto, N. et al. 1997, Nuclear Physics, A621, 467

[56] Oey, M.S. 2000, ApJ, 542, L25

[57] Pardi, M.C., Ferrini, F., Matteucci, F. 1995, ApJ, 444, 207

[58] Pipino, A., Matteucci, F., Borgani, S., Biviano, A., 2002, New Astronomy, 7, 227

[59] Rauscher, T., Hoffman, R.D., Woosley, S.E. 2002, ApJ, 576, 323

[60] Renzini, A., Ciotti, L., 1993, ApJ, 416, L49

[61] Rosenberg, A., Saviane, I., Piotto, G., Aparicio, A., 1999, AJ, 118, 2306

[62] Scalo, J.M., 1986, Fund. Cosmic Phys., 11, 1

[63] Scalo, J.M., 1998, in “The Stellar Initial Mass Function”, A.S.P. Conf. Ser. Vol. 142 p.201

[64] Schmidt, M. 1959, ApJ, 129, 243

[65] Shetrone, M.D., Cote',P., Sargent, W.L.W., 2001, ApJ, 548, 592

[66] Siess, L, Livio, M., Lattanzio, J. 2002, ApJ, 570, 329

[67] Stanford, S.A., Eisenhardt, P.R., Dickinson, M., 1998, ApJ, 492, 461

[68] Steinmetz, M., 2003, Ap\&SS, 284, 31

[69] Thielemann, F.K., Nomoto, K., Hashimoto, M., 1996, ApJ, 460, 408

[70] Tinsley, B.M. 1979, ApJ, 229, 1046

[71] Tinsley, B.M., Larson, R.B., 1979, MNRAS, 186, 503

[72] Thomas, D., Maraston, C., Bender, R., 2002, Reviews in Modern Astronomy, Vol. 15, p.219

[73] Tolstoy, E., Venn, K.A., Shetrone, M., Primas, F., Hill, V., Kaufer, A., Szeifert, T., 2003, ApJ, 125, 707

[74] Tornambé, A., Matteucci, F. 1986, MNRAS, 223, 69

[75] Trager, S. C., Faber, S.M., Worthey, G., Gonzalez, J.J., 2000, AJ, 120, 165

[76] Trager, S.C., Worthey, G., Faber, S.M., Burstein, D., Gonzalez, J.J., 1998, ApJS, 116, 1 
[77] Tsujimoto, T., Shigeyama, T., Yoshii, Y.,1999, ApJ, 519, L63

[78] Umeda, H., Nomoto, K. 2002, ApJ, 565, 385

[79] van den Hoek, L.B., Groenewegen, M.A.T., 1997, A\&AS, 123, 305

[80] Ventura, P., D’Antona, F., Mazzitelli, I., 2002, A\&A, 393, 21

[81] Vladilo, G., 2002, A\&A, 391, 407

[82] Zepf, S.E., 1997, Nature, 390, 377

[83] Whelan, J., Iben, I. Jr., 1973, ApJ, 186, 1007

[84] Woosley, S.E., Weaver, T.A., 1995, ApJS, 101, 181

[85] Worthey, G., Faber, S.M., Gonzalez, J.J., 1992, ApJ, 398, 69

[86] Wyse, R.F.G., Gilmore, G., 1992, AJ, 104, 144 